\title{
Trends and Risk Factors for Ciprofloxacin Resistance and Extended- spectrum Beta-lactamase Production in Uropathogens from Urology and Non-urology Outpatients
}

\author{
(D) Bora Özveren1, (1) Kamil Fehmi Narter2, (1) Levent Türkeri1, (1) Ahmet Şahin1 \\ 'Acıbadem Mehmet Ali Aydınlar University Faculty of Medicine, Department of Urology, Istanbul, Turkiye \\ ${ }^{2}$ Acıbadem Mehmet Ali Aydınlar University Vocational School of Health Services, Istanbul, Turkiye
}

\section{What's known on the subject? and What does the study add?}

The increased rates of antibiotic resistance of urinary pathogens influence the empirical medical management of urological infections in the outpatient setting. In this study, increased ciprofloxacin resistance and extended-spectrum beta-lactamase production were associated with being adult, male and old and isolates of E. coli and Klebsiella spp.

\begin{abstract}
Objective: This study aimed to identify the patterns and temporal changes of ciprofloxacin resistance and extended-spectrum beta-lactamase (ESBL) production in uropathogenic isolates obtained from urology and non-urology outpatients.

Materials and Methods: In this cross-sectional study, electronic data of urine culture and antimicrobial susceptibility test results of samples collected in urology and non-urology outpatient departments from 2008 to 2016 were retrospectively analysed to identify correlations between basic demographic features and clinical settings.

Results: Escherichia coli (E. coli) was the most prevalent (70\%) uropathogenic isolate in a cohort of 7.973 patients consisting of $82.8 \%$ women, $70.7 \%$ adults and $15.7 \%$ urology outpatients. Overall, resistance to ciprofloxacin was found in $16.3 \%$ of the patients. Ciprofloxacin resistance was associated with being male and old, observed more frequently in urology outpatients, detected in 19.2\% of E. coli isolates and increased to $54.5 \%$ among ESBL-producing bacterial strains $(\mathrm{p}<0.05)$. ESBL production was observed in $12 \%$ of all isolates. Increased ESBL production was associated with old age and isolates of E. coli and Klebsiella spp. ( $\mathrm{p}<0.05)$. Statistical analysis using multivariate generalised linear mixed models (mGLMMs) to assess the relationship between the outcomes predicted a significantly higher ESBL production in E. coli and Klebsiella spp. isolates and in geriatric patients. Furthermore, mGLMM analysis predicted a significantly increased likelihood of ciprofloxacin resistance in older adult male patients, especially in E. coli and Enterococcus spp. isolates. Moreover, a high rate of ESBL production was observed, reaching over 15\% in 2015 ( $<<0.05$ ). The rates of ciprofloxacin resistance remained $>10 \%$ and peaked in $2015(20.2 \%, p<0.001)$. However, in 2016, the rate of ESBL production and ciprofloxacin resistance started to decline, displaying significance only regarding the latter $(\mathrm{p}<0.05)$.

Conclusion: Empirical ciprofloxacin treatment of community-acquired urinary tract infections carries a higher risk of an unsuccessful outcome in male, geriatric and urology outpatients. Empirical antibacterial therapy for urological infections in the outpatient setting should be conducted based on patient risk profiles and contemporary local resistance data.
\end{abstract}

Keywords: Urinary tract infections, antibiotic resistance, ciprofloxacin, extended-spectrum beta-lactamases, uropathogens

\section{Introduction}

The increasing prevalence of antibiotic-resistant bacteria restricts the utility of empirical treatment of communityacquired urinary tract infections (CAUTIs). Current monitoring of the regional pattern of bacterial resistance is essential in managing appropriate treatments of urological infections. The European Association of Urology guidelines strongly recommend against using fluoroquinolones to treat uncomplicated cystitis, but stated fluoroquinolones as the first-line regimen (empiric

Correspondence: Bora Özveren MD, Acıbadem Mehmet Ali Aydınlar University Faculty of Medicine, Department of Urology, İstanbul, Turkiye Phone: +90 2166495713 E-mail: ozverenb@yahoo.com ORCID-ID: orcid.org/0000-0001-8902-7530

Received: 06.12 .2020 Accepted: 15.01.2021

Cite this article as: Özveren B, Narter KF, Türkeri L, Şahin A. Trends and Risk Factors for Ciprofloxacin Resistance and Extended-spectrum Beta-lactamase Production in Uropathogens from Urology and Non-urology Outpatients. J Urol Surg 2021;8(2):135-141.

๑Copyright 2021 by the Association of Urological Surgery / Journal of Urological Surgery published by Galenos Publishing House. 
or sensitivity-directed) of complicated UTIs, pyelonephritis, prostatitis and epididymitis/orchitis (1). If there is a lack of a coordinated strategy for judicious use of antimicrobials, quinolones may often be prescribed for the empirical treatment of CAUTIs in various clinical settings, contrary to the guidelines (2).

Remarkably high rates of fluoroquinolone resistance have been observed globally in Escherichia coli (E. coli) and Klebsiella pneumonia strains, which are common causes of healthcareassociated and community-acquired UTIs $(3,4)$. Although incidences vary by geography, multinational studies have reported fluoroquinolone-resistant $E$. coli isolates in $20 \%$ of uncomplicated and more than 50\% of complicated CAUTIs $(4,5)$.

Extended-spectrum beta-lactamases (ESBLs) are a heterogeneous group of enzymes responsible for resistance against betalactam antibiotics, which are among the most frequently used antibiotics in outpatient settings worldwide (6). ESBL-producing Enterobacteriaceae spp. commonly show cross-resistance to other groups of antibiotics, such as fluoroquinolones. The close relationship between ESBL production and ciprofloxacin (CIP) resistance is particularly troublesome because it narrows the range of alternative therapies for isolates harbouring both mechanisms at the same time (7).

In this study, the primary aim was to determine the current regional situation and temporal changes in antimicrobial resistance in common uropathogens. This study set out to explore the rates of CIP resistance and ESBL production in urinary isolates obtained from outpatients and examine the relationship between patterns of resistance with demographic features and clinical settings.

\section{Materials and Methods}

In this cross-sectional study, the electronic database of urine culture and antimicrobial susceptibility test results of samples collected in outpatient departments of Acibadem Kadiköy Hospital affiliated to Acibadem Mehmet Ali Aydinlar University (Istanbul, Turkiye) from 2008 to 2016 was analysed retrospectively. Outpatients were defined as paediatric $(<18$ years old), adult and geriatric ( $>65$ years old) individuals who visited hospital-based outpatient clinics. Urine samples were sent to the in-hospital laboratory for culture and sensitivity tests.

The inclusion criteria were as follows: any age, presentation of a urine sample to the in-hospital laboratory as an outpatient who has positive cultures for common urinary pathogens during the study period, CIP susceptibility and available ESBL activity data. All cultures were collected from outpatients with medical requests from the urology department and all other outpatient clinics from midstream urine samples, except for children aged $<2$ years old who had collections by sterile collector vials or urethral catheterisation.

This study analysed CIP susceptibility and ESBL activity data exclusively for uropathogens, namely, E. coli, Klebsiella spp., Proteus spp., Enterococcus spp., Enterobacter spp., Staphylococcus saprophyticus and "other" rare isolates. Data collected during the study period were analysed to determine the prevalence, tendency of uropathogens to CIP resistance and ESBL production and to examine risk factors among outpatient groups.

Patients were excluded from the study if they had more than one culture collected $<30$ days apart (to eliminate contamination caused by recurrent or resistant UTIs, and multiple samples were sent on a patient during treatment), urinary growth of nonuropathogenic bacterial strains and $>2$ strains simultaneously or any bacterial growth with a density $<10^{5} \mathrm{CFU} / \mathrm{mL}$ in adults and $<10^{3} \mathrm{CFU} / \mathrm{mL}$ in children whose sample was taken by urinary catheterisation.

Bacterial growth was expressed as the number of CFUs $/ \mathrm{mL}$. Identification of bacteria, antibiotic susceptibility tests and detection of ESBL production was performed by Phoenix (Becton Dickinson, USA) automated system, following the standard procedures defined by the Clinical and Laboratory Standards Institute (8).

\section{Statistical Analysis}

Statistical analyses were performed using the R Statistical Software. Descriptive statistics were used to summarise data. Binary logistic regression analyses were used for univariable comparisons. Multivariate generalised linear mixed models (mGLMMs) with logit link function were used to assess the relationship between outcomes (presence of ESBL production and CIP resistance as dependent variables) and exploratory variables (gender, age, uropathogen, outpatients and year as independent variables). The reference categories for statistical (mGLMM) analysis were "female" for gender, " $<18$ years old" for age, "others" for uropathogens, "non-urology" for outpatients and "2008" for years. A p-value $<0.05$ was considered significant.

\section{Ethics Approval}

This is a non-interventional research based on a retrospective chart review of electronic data. Approval for the study protocol was obtained from the Acibadem MAA University Institutional Ethics Committee (no: 2020-12/4). The study was conducted according to the criteria set by the Declaration of Helsinki. Waiver of patient consent was approved by local institutional ethics committee. All data were analysed anonymously, and the waiver of informed consent did not and will not have adverse effect on the rights and health of the patients. 


\section{Results}

Among 7973 urinary isolates, $82.8 \%$ belonged to female patients and $70.7 \%$ belonged to adult and geriatric patients. Isolates from the urology outpatients comprised $15.7 \%$ of the cohort. E. coli was the most prevalent (70\%) uropathogen isolated, followed by Klebsiella spp. (10.8\%), Enterococcus spp. (7.5\%), Proteus spp. (3.7\%), Enterobacter spp. (2\%), Staphylococcus saprophyticus (1.2\%) and others (4.9\%). Table 1 displays the summary statistics of the cohort and uropathogens.

Overall, ESBL production was observed in $12 \%$ and CIP resistance was found in $16.3 \%$ of the isolates. The rate of CIP resistance was 54.5\% among all ESBL-positive uropathogens and 58.4\% in ESBL-positive E. coli isolates, whereas it remained at $11.1 \%$ among ESBL-negative isolates.

\section{Patterns of ESBL Activity}

ESBL production was markedly higher in the geriatric group $(p<0.001)$. No difference was found in the rate of ESBL activity related to gender or between isolates from urology and nonurology outpatient clinics ( $p>0.05)$. ESBL production was significantly increased (15\%) in E. coli and Klebsiella spp. isolates $(p<0.05)$ (Table 2). The rates of ESBL production among isolated uropathogens throughout the study period are given in Table 2 , where a high rate of ESBL production reaching $>15 \%$ in 2014 and 2015 stands out $(p<0.05)$ (Figure 1).

The mGLMM analysis was performed to assess a range of factors on ESBL production, and the final model was found significant for prediction $(F=19.236, p<0.001)$ (Table 3). The statistical

\begin{tabular}{|l|l|l|}
\hline $\begin{array}{l}\text { Table 1. Patient demography and distribution of } \\
\text { microorganisms of interest isolated from urine samples }\end{array}$ \\
\hline \multirow{4}{*}{ Gender } & & n (\%) \\
\hline \multirow{4}{*}{ Age } & Female & $6604(82.8)$ \\
\cline { 2 - 3 } & Male & $1369(17.2)$ \\
\hline \multirow{4}{*}{ Outpatients } & Paediatric (0-17) & $2337(29.3)$ \\
\cline { 2 - 3 } & Adult (18-65) & $4654(58.4)$ \\
\cline { 2 - 3 } & Geriatric (>65) & $982(12.3)$ \\
\hline \multirow{5}{*}{ Uropathogens } & Urology & $1252(15.7)$ \\
\cline { 2 - 3 } & Non-urology & $6721(84.3)$ \\
\cline { 2 - 3 } & Escherichia coli & $5580(70)$ \\
\cline { 2 - 3 } & Klebsiella spp. & $864(10.8)$ \\
\cline { 2 - 3 } & Proteus spp. & $292(3.7)$ \\
\cline { 2 - 3 } & Enterococcus spp. & $599(7.5)$ \\
\cline { 2 - 3 } & Enterobacter spp. & $156(2)$ \\
\cline { 2 - 3 } & Staphylococcus saprophyticus & $95(1.2)$ \\
\hline & Others* & $387(4.9)$ \\
\hline
\end{tabular}

*Rare isolates referred as "others" include Pseudomonas aeruginosa, Morganella morganii, Serratia marcescens and Streptococcus agalactiae analysis revealed that the risk of ESBL production of isolates was significantly higher in patients aged $>65$ years [odds ratio (OR) (95\% confidence interval (Cl) $2.095(1.702,2.580), p<0.001]$. The risk of ESBL positivity was lower in 2012 [OR $(95 \% \mathrm{Cl}) 0.624$ $(0.428,0.909), p=0.014]$ (Table 3). Among uropathogens, E. coli and Klebsiella spp. were associated with higher prevalence of ESBL production $(p<0.001)$. Further analysis showed that the probability of ESBL production remained significantly high from 2014 through 2016.

\section{Patterns of CIP Resistance}

Male sex and old age among patient groups were significant factors for CIP resistance $(p<0.05)$. CIP resistance was more often observed in isolates from urology than those delivered from non-urology outpatient clinics (19.6\% vs $15.7 \%, p<0.05)$. The statistical analysis also detected a remarkably high (19.2\%) level of CIP resistance in $E$. coli isolates $(p<0.001)$ (Table 2).

During the study period, the rates of CIP resistance constantly remained $>10 \%$. From 2012 to 2015, a continual rise was noted, reaching the highest rate in $2015(20.2 \%, p<0.001)$. However, in 2016 , the rate of CIP resistance significantly decreased to $12.7 \%$ $(p<0.05)$ (Figure 1).

The mGLMM analysis was performed to assess the effects of numerous factors on CIP resistance, and the obtained model was significant $(F=30.810, p<0.001)$. The results established a relationship of elevated risk of CIP resistance with male sex [OR $(95 \% \mathrm{Cl}) 1.357(1.140,1.616), p=0.001]$ and increased age. The probability of CIP resistance was higher in adults (18-65 years) [OR $(95 \% \mathrm{Cl}) 2.013(1.685,2.405), \mathrm{p}<0.001]$ and the highest in the geriatric group [OR $(95 \% \mathrm{Cl}) 7.544(6.151,9.254), \mathrm{p}<0.001]$.

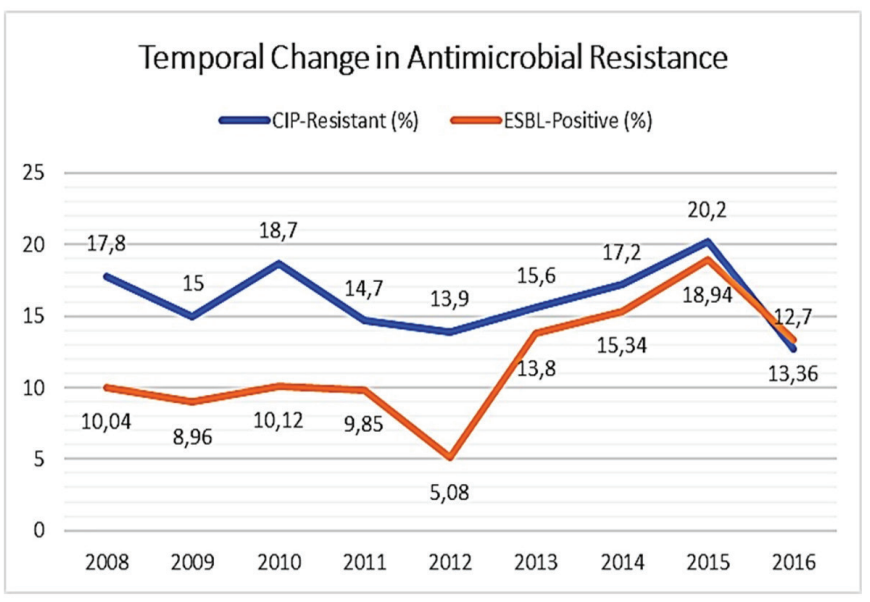

Figure 1. Frequency of ciprofloxacin resistance and extended-spectrum beta-lactamase production of uropathogens isolated from urine samples of outpatients evaluated from 2008 to 2016 For ciprofloxacin resistance: $\mathrm{p}<0.05$ in 2015 and 2016

For extended-spectrum beta-lactamase production: $p<0.05$ in 2008, 2009, 2011, 2012, 2014 and 2015 
The likelihood of CIP resistance was significantly increased in isolates of $E$. coli and Enterobacter spp. $(p<0.001)$. The relative declines in the risk of CIP resistance in 2013 [OR (95\% CI) 0.766 $(0.597,0.983), p=0.036]$ and 2016 [OR (95\% Cl) 0.631 (0.488, $0.815), p<0.001]$ were significant (Table 3 ).

\section{Discussion}

This study was carried out to determine the recent local prevalence of uropathogens and antimicrobial susceptibility patterns by assessing CIP resistance and ESBL activity in isolates. Data collected from urology and non-urology outpatients in

Table 2. ESBL production and CIP resistance status of urinary isolates according to patient features, bacterial strains and years

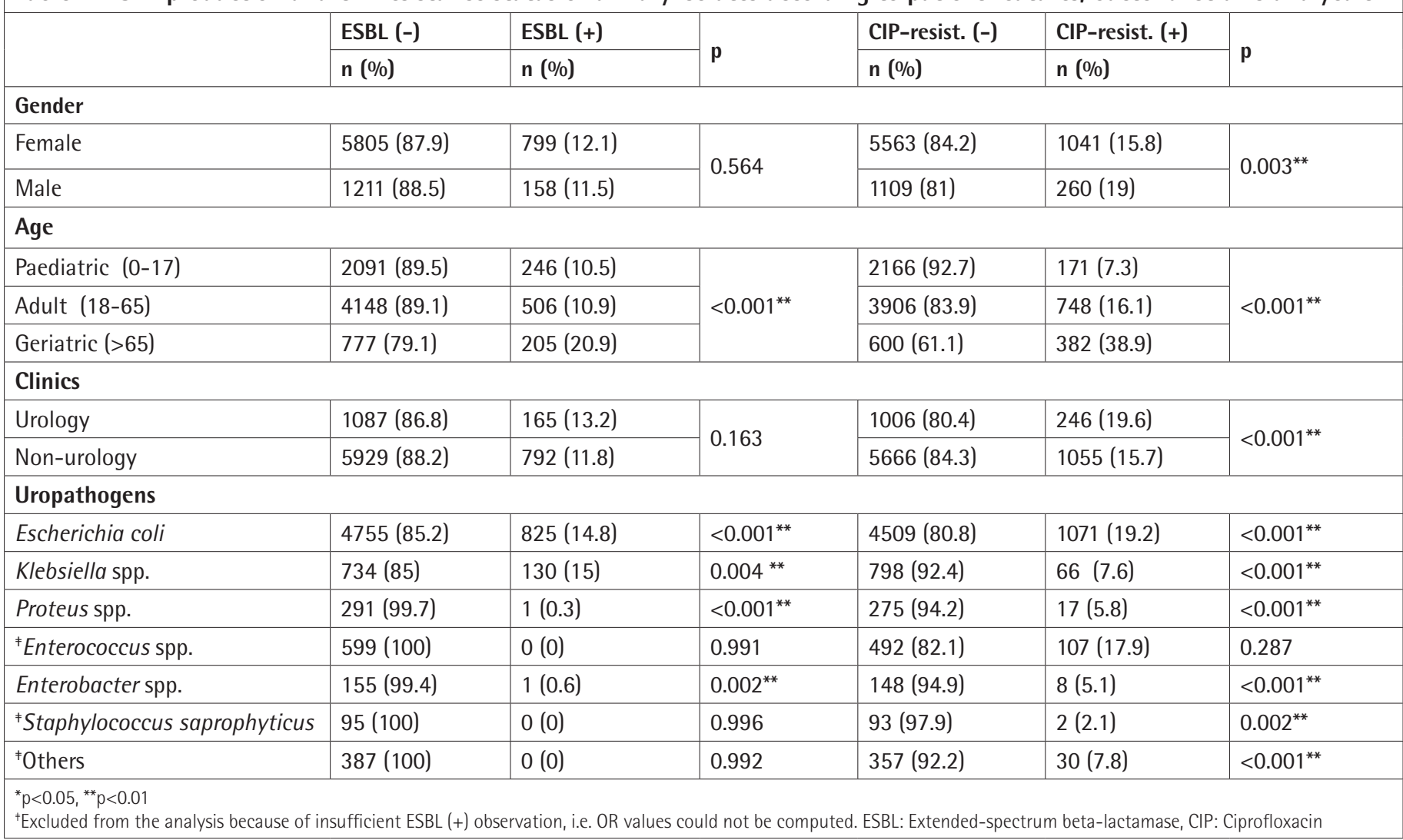

Table 3. Multivariate generalised linear mixed model analysis of the relationship between the outcomes (presence of ESBL production and CIP resistance) and exploratory variables (gender, age, uropathogen, outpatient clinic and year)

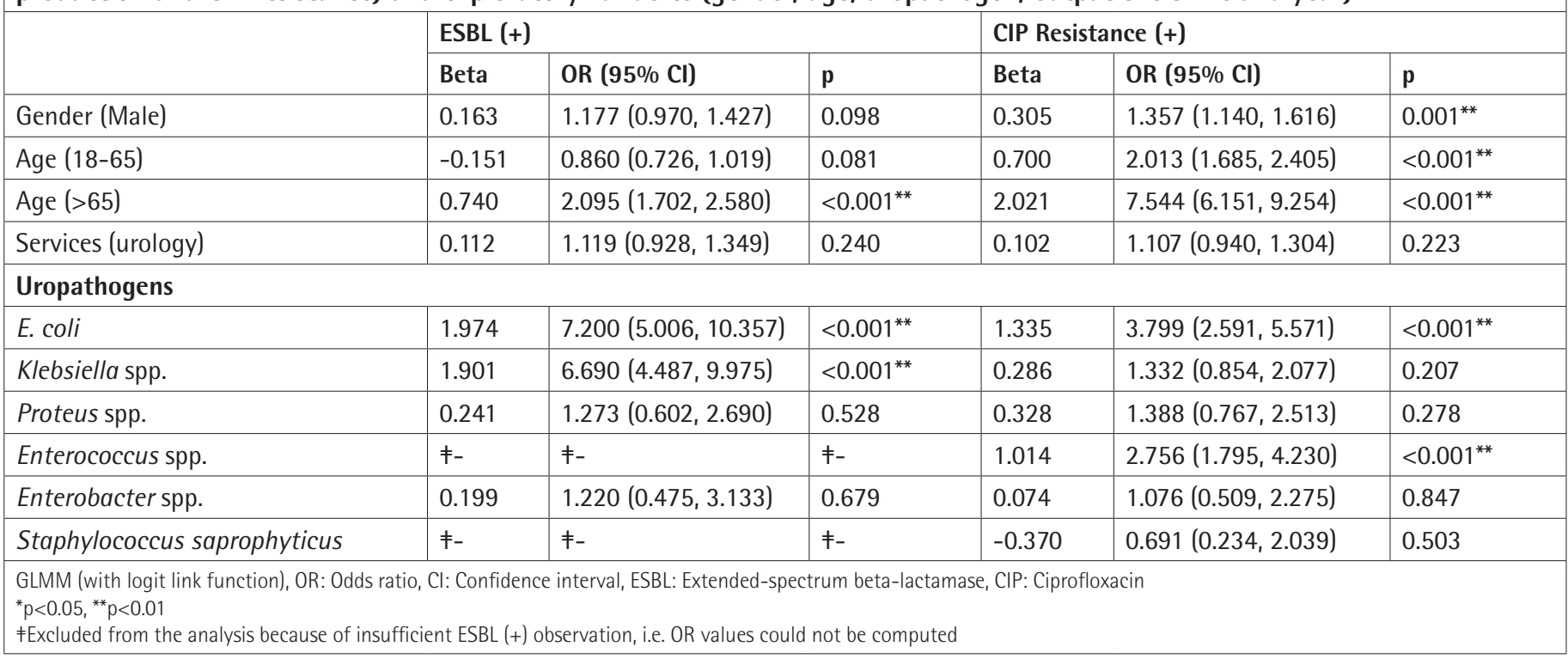


a 9-year interval were retrospectively examined. This study confirms that $E$. coli is the predominant uropathogen isolated in CAUTIs. The frequency rate $(70 \%)$ was similar to the rate observed in other prevalence studies that examined data from patients clinically diagnosed with UTI.

Recent multinational, prospective surveillance studies in Europe established an E. coli prevalence of 74\%-76.7\% in women with acute uncomplicated UTI $(9,10)$. International studies that are methodologically comparable to the present study have reported $E$. coli prevalence of $56.8 \%-70.4 \%(11,12)$. A previous study conducted in Turkey reported that $E$. coli is the causative agent in $90 \%$ of the uncomplicated CAUTIs and $78 \%$ of the complicated CAUTIs (13). Differences regarding prevalence could be attributed to a broader selection of outpatients with uncomplicated and complicated UTIs in the current cohort. The microbiology of complicated UTI is characterised by a greater spectrum of bacterial strains and an increased likelihood of antimicrobial resistance compared with acute uncomplicated UTI (14).

\section{Resistance Patterns}

The pattern of CIP resistance was the main focus in this study owing to its key role in the management of complicated urological infections. We identified an overall CIP resistance rate of $16.3 \%$ among common uropathogens, which increased to 19.2\% among E. coli isolates and 54.5\% among ESBL-producing bacterial strains. Surveillance studies have demonstrated widely fluctuating rates of CIP resistance in different geographical areas (4). A large multicentre surveillance study, with a similar methodology to this study, reported a $5.5 \%$ of CIP resistance rate in North America (15). A meta-analysis of observational studies revealed an estimated pooled CIP resistance of 27\% in CAUTIs caused by E. coli (5). For uncomplicated CAUTIs, rates of CIP resistance ranged from $0 \%$ to $14.7 \%$ in Europe, with the lowest in Nordic countries and Austria and highest in Portugal and Spain (9). In Turkey, results from earlier studies demonstrated a 25\%-38\% frequency of CIP resistance among urinary pathogens $(13,16)$. Overuse or misuse of antibiotics is known to propagate bacterial multidrug resistance. A positive correlation was found between widespread prescription of quinolones and antibiotic resistance, limiting their effectiveness in the treatment of UTIs. Moreover, in communities, frequent use of prescription drugs without medical advice may contribute to increasing bacterial resistance (2).

ESBLs frequently carry resistance genes for additional antibiotic classes including fluoroquinolones (17). Hence, ESBL activity of Gram-negative bacteria may be viewed as a surrogate to multidrug resistance. ESBL production ranges widely from $2.6 \%$ to $100 \%$ in various geographical areas, highest in the Asia-Pacific region and moderate to low in Europe and North
America (4). A study from Turkey reported a 17.4\% rate of ESBLproducing $E$. coli in adults with CAUTI (15). In the present study the level of ESBL production in E. coli isolates observed was $14.8 \%$. As one of the significant findings emerging from our data, among ESBL-positive isolates, the CIP resistance coexisted in $54.5 \%$, in contrast to $11.1 \%$ among ESBL-negative isolates. Studies have demonstrated that CIP resistance is more common in ESBL-positive uropathogenic E. coli isolates in CAUTIs $(7,16)$. ESBL production is also higher in $E$. coli isolates in complicated CAUTIs than in uncomplicated cases $(15,18)$. Taken together, these data suggest a close correlation between ESBL production and fluoroquinolone resistance in uropathogenic bacteria. In regions where ESBL-producing Gram-negative communityacquired uropathogens are common, enhanced efforts for accurate determination of ESBL activity in combination with antibiotic sensitivity is warranted, along with restriction of fluoroquinolones in empirical treatments.

In the present study, isolates from older (geriatric > adult), male, adult urology outpatients were related to higher CIP resistance. Additionally, isolates of $E$. coli, leading pathogen in community-onset urological infections, were more likely to exhibit CIP resistance and ESBL production. A higher risk of ESBL production was further related to the older age ( $>65$ years) of outpatients, which is known as one of the common risk factors for community-onset ESBL-producing E. coli or Klebsiella spp. infections $(3,18-22)$. The incidence of UTI is increased in elderly patients owing to their immune status and aging-related physiological and anatomical changes. Elderly patients are more likely to be immunocompromised, have co-morbidities and are hospitalised more often than younger patients. Such conditions expose them to frequent or high consumption of antimicrobial drugs, which brings about an increment of resistance to antimicrobial agents (20-23).

Assessment of regional, population-based resistance patterns data and patient-specific risk factors data is key to establishing country-specific guidelines on empirical antibiotic treatment recommendations (24).

In the present study, the $19.2 \%$ CIP resistance rate in E. coli isolates raises a concern regarding the clinically meaningful susceptibility threshold for fluoroquinolones in CAUTIs. Statistical modelling of our results can infer that an empirical CIP treatment of CAUTI carries a higher risk of an unsuccessful outcome in a male, geriatric, urology outpatient.

\section{Temporal Changes in Antimicrobial Resistance}

We observed an increasing level of CIP resistance among uropathogens during the study period with a peak (20.2\%) in 2015. Surprisingly, a significant decrease was noted in CIP resistance in 2016. Likewise, ESBL production remained stable at approximately 10\% until 2013 and thereafter increased to 
20\% in 2015. A brief decrease in ESBL production occurred in 2016, albeit without significance. The present results provided additional and contemporary evidence regarding the persistent problem of antimicrobial resistance in CAUTIs, which represents a challenge to urology practice. Comprehensive reviews indicate a continuous, worldwide increase of antimicrobial resistance. In southern European countries as well as in the USA, a gradual increase in the resistance of $E$. coli to fluoroquinolones has been reported (24). In Switzerland, an analysis of urinary E. coli specimens obtained from 1997 to 2007 found an increasing trend in CIP resistance from $1.8 \%$ to $15.9 \%$ (25). A gradual rise in resistance of $E$. coli strains to CIP from $8 \%$ to $11 \%$ in 2009-2011 is noted in Australia (26). Studies on antimicrobial resistance have revealed that irrational prescription habits and high consumption of fluoroquinolones lead to the dissemination of quinolone resistance in the community $(22,24)$. The high quinolone resistance in our region may be due to increased fluoroquinolone consumption over the years. In 2013, a "Rational Drug Use National Action Plan" was issued by the Turkish Ministry of Health, implementing several integrated interventions to survey, contain and prevent antimicrobial resistance emergence and spread. It could be argued that the significant decrease in CIP resistance in the last year of the study period may be attributed to the positive result of the plan; however, further research should be undertaken to investigate the consequences of this initiative.

\section{Study Limitations}

This study has some limitations. First, hospital and laboratorybased surveillance data obtained from a single centre may overestimate the rates of antibiotic resistance. Uncomplicated UTIs in the outpatient setting may be treated empirically without sending a urine culture sample to the laboratory. Cultures are usually performed if the patient fails to respond to treatment, has recurrent episodes of UTI or has complicated UTI. Second, data are retrospectively analysed, and the investigation is limited by the lack of uniform clinical information on previous antibiotic treatment, previous hospitalisations and interventions, whether urine samples came from patients with asymptomatic bacteriuria, uncomplicated or complicated UTIs. Since our data lacked information on symptomatology or clinical history of patients, we excluded uncommon urinary isolates to omit cases that may not have represented CAUTIs. The analysis of in vitro microbiological data alone may have altered uropathogen prevalence.

The strengths of our study are the large sample size, use of only common community-acquired uropathogenic isolates to avoid selection bias and the very stringent classification of electronic surveillance data.

\section{Conclusion}

It is essential to know the current, local bacteriological environment and resistance patterns as well as risk factors to guide the physicians in choosing the appropriate antibiotic treatment for infections. The increased rates of CIP resistance and ESBL production of urinary pathogens undeniably influence the medical management of urological infections in the outpatient setting. Determined efforts regarding a comprehensive policy and detailed action plans on prudent use of all antibiotics, including CIP should be developed and enforced by regional multidisciplinary teams.

\section{Ethics}

Ethics Committee Approval: Approval for the study protocol was obtained from the Acibadem MAA University Institutional Ethics Committee (no: 2020-12/4).

Informed Consent: Waiver of patient consent was approved by local institutional ethics committee. All data were analysed anonymously, and the waiver of informed consent did not and will not have adverse effect on the rights and health of the patients.

Peer-review: Internally peer-reviewed.

\section{Authorship Contributions}

Surgical and Medical Practices: B.Ö., K.F.N., L.T., A.Ş., Concept: B.Ö., K.F.N., L.T., A.Ş., Design: B.Ö., K.F.N., L.T., A.Ş., Data Collection or Processing: B.Ö., K.F.N., Analysis or Interpretation: B.Ö., K.F.N., L.T., A.Ş., Literature Search: B.Ö., Writing: B.Ö.

Conflict of Interest: No conflict of interest was declared by the authors.

Financial Disclosure: The authors declare that they have no relevant financial.

\section{References}

1. Naber KG, Bergman B, Bishop MC, Bjerklund-Johansen $T E$, Botto $H$, Lobel $B$, Jinenez Cruz F, Selvaggi FP; Urinary Tract Infection (UTI) Working Group of the Health Care Office (HCO) of the European Association of Urology (EAU). EAU guidelines for the management of urinary and male genital tract infections. Urinary Tract Infection (UTI) Working Group of the Health Care Office (HCO) of the European Association of Urology (EAU). Eur Urol 2001;40:576-588.

2. Morgan DJ, Okeke IN, Laxminarayan R, Perencevich EN, Weisenberg S. Nonprescription antimicrobial use worldwide: a systematic review. Lancet Infect Dis 2011;11:692-701.

3. Mazzariol A, Bazaj A, Cornaglia G. Multi-drug-resistant Gram-negative bacteria causing urinary tract infections: a review. J Chemother 2017;29(Suppl 1):2-9.

4. Dalhoff A. Global fluoroquinolone resistance epidemiology and implictions for clinical use. Interdiscip Perspect Infect Dis 2012;2012:976273. 
5. Fasugba O, Gardner A, Mitchell BG, Mnatzaganian G. Ciprofloxacin resistance in community- and hospital-acquired Escherichia coli urinary tract infections: a systematic review and meta-analysis of observational studies. BMC Infect Dis 2015;15:545.

6. Karlowsky JA, Lagacé-Wiens PR, Simner PJ, DeCorby MR, Adam HJ, Walkty $A$, Hoban DJ, Zhanel GG. Antimicrobial resistance in urinary tract pathogens in Canada from 2007 to 2009: CANWARD surveillance study. Antimicrob Agents Chemother 2011;55:3169-3175.

7. Pitout JD, Nordmann $P$, Laupland KB, Poirel L. Emergence of Enterobacteriaceae producing extended-spectrum beta-lactamases (ESBLs) in the community. J Antimicrob Chemother 2005;56:52-59.

8. Clinical and Laboratory Standards Institute (CLSI). Performance standards for antimicrobial disk susceptibility tests; Approved standard-Ninth edition. CLSI document M2-A9. CLSI, Wayne, PA, USA: 2006.

9. Kahlmeter G, Poulsen HO. Antimicrobial susceptibility of Escherichia coli from community-acquired urinary tract infections in Europe: the ECO-SENS study revisited. Int J Antimicrob Agents 2012;39:45-51.

10. Naber KG, Schito G, Botto H, Palou J, Mazzei T. Surveillance study in Europe and Brazil on clinical aspects and Antimicrobial Resistance Epidemiology in Females with Cystitis (ARESC): implications for empiric therapy. Eur Urol 2008;54:1164-1175

11. Sanchez GV, Master RN, Karlowsky JA, Bordon JM. In vitro antimicrobial resistance of urinary Escherichia coli isolates among U.S. outpatients from 2000 to 2010. Antimicrob Agents Chemother 2012;56:2181-2183.

12. Cullen IM, Manecksha RP, McCullagh E, Ahmad $S$, O'Kelly F, Flynn $R$, McDermott TE, Murphy P, Grainger R, Fennell JP, Thornhill JA. An 11year analysis of the prevalent uropathogens and the changing pattern of Escherichia coli antibiotic resistance in 38,530 community urinary tract infections, Dublin 1999-2009. Ir J Med Sci 2013;182:81-89.

13. Arslan H, Azap OK, Ergönül O, Timurkaynak F; Urinary Tract Infection Study Group. Risk factors for ciprofloxacin resistance among Escherichia coli strains isolated from community-acquired urinary tract infections in Turkey. J Antimicrob Chemother 2005;56:914-918.

14. Nicolle LE. A practical guide to antimicrobial management of complicated urinary tract infection. Drugs Aging 2001;18:243-254.

15. Zhanel GG, Hisanaga TL, Laing NM, DeCorby MR, Nichol KA, Palatnik LP, Johnson J, Noreddin A, Harding GK, Nicolle LE, Hoban DJ; NAUTICA Group. Antibiotic resistance in outpatient urinary isolates: final results from the North American Urinary Tract Infection Collaborative Alliance (NAUTICA). Int J Antimicrob Agents 2005;26:380-388.
16. Azap OK, Arslan H, Serefhanoğlu K, Colakoğlu S, Erdoğan H, Timurkaynak F, Senger SS. Risk factors for extended-spectrum beta-lactamase positivity in uropathogenic Escherichia coli isolated from community-acquired urinary tract infections. Clin Microbiol Infect 2010;16:147-151.

17. Bonkat G, Müller G, Braissant $\mathrm{O}$, Frei $\mathrm{R}$, Tschudin-Suter $\mathrm{S}$, Rieken $\mathrm{M}$, Wyler $\mathrm{S}$, Gasser TC, Bachmann A, Widmer AF. Increasing prevalence of ciprofloxacin resistance in extended-spectrum- $\beta$-lactamase-producing Escherichia coli urinary isolates. World J Urol 2013;31:1427-1432.

18. Oteo J, Pérez-Vázquez $M$, Campos J. Extended-spectrum [beta]-lactamase producing Escherichia coli: changing epidemiology and clinical impact. Curr Opin Infect Dis 2010;23:320-326.

19. Sahm DF, Thornsberry C, Mayfield DC, Jones ME, Karlowsky JA. Multidrugresistant urinary tract isolates of Escherichia coli: prevalence and patient demographics in the United States in 2000. Antimicrob Agents Chemother 2001;45:1402-1406

20. Killgore KM, March KL, Guglielmo BJ. Risk factors for community-acquired ciprofloxacin-resistant Escherichia coli urinary tract infection. Ann Pharmacother 2004;38:1148-1152.

21. Koksal E, Tulek N, Sonmezer MC, Temocin F, Bulut C, Hatipoglu C, Erdinc FS, Ertem $\mathrm{G}$. Investigation of risk factors for community-acquired urinary tract infections caused by extended-spectrum beta-lactamase Escherichia coli and Klebsiella species. Investig Clin Urol 2019;60:46-53.

22. Dalhoff A. Resistance surveillance studies: a multifaceted problem--the fluoroquinolone example. Infection 2012;40:239-262.

23. Elias C, Moja L, Mertz D, Loeb M, Forte G, Magrini N. Guideline recommendations and antimicrobial resistance: the need for a change. BMJ Open 2017;7:016264.

24. Gupta K, Hooton TM, Stamm WE. Increasing antimicrobial resistance and the management of uncomplicated community-acquired urinary tract infections. Ann Intern Med 2001;135:41-50.

25. Blaettler L, Mertz D, Frei R, Elzi L, Widmer AF, Battegay M, Flückiger U. Secular trend and risk factors for antimicrobial resistance in Escherichia coli isolates in Switzerland 1997-2007. Infection 2009;37:534-539.

26. Fasugba O, Mitchell BG, Mnatzaganian G, Das A, Collignon P, Gardner A. Five-Year Antimicrobial Resistance Patterns of Urinary Escherichia coli at an Australian Tertiary Hospital: Time Series Analyses of Prevalence Data. PLoS One 2016;11:0164306. 